\title{
Myocilin and optineurin coding variants in Hispanics of Mexican descent with POAG
}

\author{
Kristin K McDonald ${ }^{1}$, Karen Abramson ${ }^{1}$, Marco A Beltran ${ }^{2}$, Maria G Ramirez ${ }^{3}$, Miguel Alvarez ${ }^{3}$, \\ Alice Ventura ${ }^{4}$, Cecilia Santiago-Turla ${ }^{4}$, Silke Schmidt ${ }^{1}$, Michael A Hauser ${ }^{1,4}$ and R Rand Allingham ${ }^{1,4}$ \\ Coding variants in both myocilin (MYOC) and optineurin (OPTN) are reported risk factors for primary open-angle glaucoma \\ (POAG) in many populations. This study investigated the contribution of MYOC and OPTN coding variants in Hispanics of \\ Mexican descent with and without POAG. We conducted a case-control study of unrelated POAG cases and nonglaucomatous \\ controls in a population of Hispanics of Mexican descent. Ascertainment criteria for POAG included the presence of \\ glaucomatous optic neuropathy with associated visual field loss and the absence of secondary causes of glaucoma. Controls had \\ normal optic nerves, visual fields and intraocular pressure. All coding exons of $M Y O C$ and OPTN were sequenced. The data set \\ consisted of 88 POAG cases and 93 controls. A novel nonsynonymous coding variant (R7H) in the first exon of MYOC was \\ identified. Other identified variants in $M Y O C$ and $O P T N$ have been previously described and do not seem to contribute to POAG \\ risk. This is the first comprehensive study of $M Y O C$ and $O P T N$ in Hispanics of Mexican descent with POAG. Neither MYOC nor \\ OPTN sequence variants seem to have a major role in the etiology of POAG in this population.
}

Journal of Human Genetics (2010) 55, 697-700; doi:10.1038/jhg.2010.91; published online 29 July 2010

Keywords: coding variants; myocilin; optineurin; primary open-angle glaucoma

\section{INTRODUCTION}

Glaucoma is defined as the progressive, irreversible loss of retinal ganglion cells. Primary open-angle glaucoma (POAG) is the most common form of glaucoma. POAG is a complex disorder with known genetic contributions that is often associated with elevated intraocular pressure (IOP). ${ }^{1}$ POAG is responsible for more than half of all cases of glaucoma in the world and for blindness in more than 3.3 million people. ${ }^{2,3}$ The number of people who are affected by POAG is steadily continuing to increase, and it is expected to be the cause of blindness in more than 4.4 million people globally by the year 2020.4

Individuals of Hispanic descent are at a high risk of developing POAG. Two major population-based studies focusing on ophthalmic disease and vision loss of Hispanics in southwestern United States found that POAG is highly prevalent in Hispanics. ${ }^{6,7}$ These studies reported that the prevalence of POAG in Hispanics over the age of 40 years exceeded $4 \%$ and over the age of 80 years approached $20 \%$, which is even higher than the reported prevalence in African Ameri cans. $^{2}$ The clinical phenotype also differed between Hispanics and other US populations. In all, $\sim 80 \%$ of Hispanics with POAG were found to have normal IOP on initial screening, as opposed to Caucasian and African American POAG patients, of whom the majority have elevated IOP. ${ }^{2,6,7}$ The high frequency of normal IOP on initial screening in the Hispanic population is shared by the
Japanese population and may represent underlying genetic and environmental differences between populations. ${ }^{8}$

Variants in myocilin (MYOC) and optineurin (OPTN) have been associated with risk for developing POAG. ${ }^{9-12}$ MYOC variants are found in $3-5 \%$ of POAG patients and are associated with an earlyonset, high-tension form of open-angle glaucoma. ${ }^{11,13-15}$ OPTN variants seem to be associated in patients with POAG and normal IOP. ${ }^{12,16}$ Little is known about the genetic etiology of POAG in the Hispanic population. Although MYOC and OPTN have been sequenced in Brazilian and Spanish populations, the role of these genes in POAG in Hispanics of southwestern United States has not been reported to date. ${ }^{17-19}$ In this paper we report the contribution of MYOC and OPTN coding variants to POAG risk in a data set of Hispanics of Mexican descent.

\section{MATERIALS AND METHODS}

Subject ascertainment

This study adhered to the tenets of the Declaration of Helsinki. Informed consent was obtained from all participating individuals after explanation of the nature and risks of the study. The research project was reviewed and approved by the institutional review board of Duke University Medical Center (Durham, NC, USA). Subjects for this study were recruited from eye clinics located in Sonora, Mexico and Nogales, Arizona, and described themselves as being of

${ }^{1}$ Center for Human Genetics, Duke University Medical Center, Durham, NC, USA; ${ }^{2}$ Clinica Oftalmologica, Hermosillo, Sonora, Mexico; ${ }^{3}$ Oftalmologos Asociados, Nogales, Sonora, Mexico and ${ }^{4}$ Department of Ophthalmology, Duke University Eye Center, Duke University Medical Center, Durham, NC, USA 
Hispanic descent, with both parents of Mexican ancestry. These individuals are sometimes termed Mestizos (individuals of mixed European and North American indigenous ancestry). Study subjects include individuals diagnosed with POAG, as well as unaffected spouses and other unaffected individuals.

POAG cases were unrelated and met the following inclusion criteria: (1) glaucomatous optic nerve damage in both eyes and (2) glaucomatous visual field defects in at least one eye. Glaucomatous optic nerve damage was defined as being present when two or more of the following criteria were identified: (a) vertical or horizontal cup/disc ratio $\geqslant 0.7$; (b) superior or inferior neuroretinal rim cup/disc ratio $\leqslant 0.1$; (c) focal notching of the superior or inferior neuroretinal rim; (d) asymmetry of the cup/disc ratio $\geqslant 0.2$ without asymmetric refraction; and/or (e) optic disc hemorrhage. IOP was recorded but was not used as an inclusion criterion. Visual fields were performed using standard automated perimetry. ${ }^{4}$ In addition, all cases had open angles by gonioscopy. IOP measurements $(\mathrm{mm} \mathrm{Hg})$ were taken by applanation tonometry. The highest IOP measurements used were those obtained by ophthalmologists at the time of examination. The patients were in many cases indigent and were seen on one occasion by the study ophthalmologists; no past medical history was obtained for these individuals because of the potential for variability in the manner in which IOP measurements were taken at other locations. However, for those individuals who were regular patients of the study ophthalmologists, the highest IOP measurement was the highest IOP seen by the coauthors over time. IOP measurements were not taken for patients who had ocular surgery before their examination.

Exclusion criteria included other identifiable forms of glaucoma due to or associated with exfoliation syndrome, pigmentary dispersion syndrome or traumatic glaucoma. The controls used for this study were well matched to cases by ethnicity, and met the following criteria: (1) IOP without treatment $<22 \mathrm{~mm} \mathrm{Hg}$; (2) no evidence of glaucomatous optic nerve disease; and (3) normal automated visual field testing (Humphrey SITA standard or equivalent). All examiners are board-certified ophthalmologists. All clinical data, including visual field tests and optic nerve photos, were reviewed by a board-certified glaucoma fellowship-trained ophthalmologist (RRA). Blood samples for DNA extraction were obtained from a total of 88 POAG cases and 93 POAG controls and were processed and stored at the DNA repository at the Center for Human Genetics, Duke University Medical Center. Mean IOP for cases was calculated by averaging the highest recorded IOP of both eyes where available, thereafter averaging all cases. Mean IOP for controls was calculated by averaging the IOP of both eyes at ascertainment, thereafter averaging all controls.

\section{DNA analysis}

Genomic DNA was extracted from peripheral blood by standard techniques (Gentra, Minneapolis, MN, USA). Primers flanking each exon, 1-3 in MYOC and 1-16 in OPTN, were designed with Primer3 Plus software (Humana Press, Totowa, NJ, USA) and are listed in Table $1 .^{20}$ All sequencing procedures were performed using conditions that were previously described. ${ }^{21}$ All suspected variations were confirmed by bidirectional sequencing. Sequencing was initially performed using cases only. Amplicons containing any nonsynonymous coding changes or variants of uncertain pathogenicity in cases were then sequenced in the control data set.

\section{Statistical analysis}

Sequencing data were examined using Sequencher 4.9 software (Gene Codes Corporation, Ann Arbor, MI, USA) and were analyzed as described previously. ${ }^{22}$ Briefly, allele frequencies for each coding and intronic variant in POAG cases and controls were compared using a two-sided Fisher's exact test using SAS/Genetics version 9.1 (SAS Institute, Cary, NC, USA). A $P$-value of $<0.05$ was considered statistically significant. The 176 POAG case chromosomes sequenced in this study are sufficient to detect over $99.9 \%$ of single-nucleotide polymorphisms with a minimum allele frequency of $5 \% .{ }^{23}$

\section{RESULTS}

The mean age of onset for 88 POAG cases and age of exam for 93 controls were 62.4 (s.d. \pm 11.2 ) and 62.7 (s.d. \pm 8.3 ) years, respectively. In all, $52 \%$ of POAG cases and $66 \%$ of POAG controls were female. The mean highest recorded IOP for controls was $13.8 \pm 2.7 \mathrm{~mm} \mathrm{Hg}$, whereas that of cases was significantly higher at $19.0 \pm 6.5 \mathrm{~mm} \mathrm{Hg}$

Table 1 PCR primers for sequencing genomic DNA

\begin{tabular}{|c|c|c|c|c|}
\hline & Exon & PCR forward primer sequence & $P C R$ reverse primer sequence & $\begin{array}{c}\text { PCR product } \\
\text { size }(b p)\end{array}$ \\
\hline \multirow[t]{6}{*}{ MYOC } & $1 a$ & ATCTTGCTGGCAGCGTGAA & TCTCTGGTTTGGGTTTCC & 614 \\
\hline & $1 b$ & GACAGCTCAGCTCAGGAAGG & GAAGGTGATCGCTGTGCTTT & 663 \\
\hline & 2 & AGCAAAGACAGGGTTTCACC & AGGGCTTTGTTAGGGAAAGG & 555 \\
\hline & $3 a$ & TGCGATAACTGAGGCGTAGA & GCCTCATCGGTGCTGTAAAT & 663 \\
\hline & $3 b$ & GTCCAGAACTGTCATAAGA & CGCCCTCAGACTACAATTCC & 679 \\
\hline & $3 c$ & GCCTGGGACAACTTGAACAT & CAGGCACAAGCCTCTCAGTT & 719 \\
\hline \multirow[t]{16}{*}{ OPTN } & 1 & CGGACAGCGAGGGTGGGTA & CAGGACCCGCCGAGGCTT & 554 \\
\hline & 2 & AAGCAGAGTGGGGATTTACTCA & TTCCCATGCAAATCTTCAAA & 500 \\
\hline & 3 & ССССАТТТСССАААТССТТА & GAGGCAGCTGAGAGGTTGAT & 633 \\
\hline & 4 & TAAGTATTAGCAATCGCCAA & AGTGCAAAGGGATGGCATTT & 340 \\
\hline & 5 & CATCAGATCAAGTCCACTTT & GGAGTCTAGACACGTAAGAT & 340 \\
\hline & 6 & ATGGTGCCCAGCCTTAGTTT & CAATCCTTGGCTTGTGTTGA & 340 \\
\hline & 7 & CATCTGAATGTTTGGAAGCT & TATTCTGGAAAGATCCTGGT & 340 \\
\hline & 8 & ATACTGAACAGGGCATTGTC & GTGGTTGCACAATCCTGGAA & 300 \\
\hline & 9 & GATCCTTTATCCCAATTGTA & TTGAATTCAGTTGCTGGACT & 282 \\
\hline & 10 & TTGATTCACCAGCCAGTCTT & GCTCACACATTAACTGGAAC & 400 \\
\hline & 11 & TGCATTCATAAACCCTACAG & TAGGACTCCTTCAGATAAGT & 400 \\
\hline & 12 & TTGAGAGTAAGAAATGCTAG & GATTTAGTGAAGGATTCATG & 340 \\
\hline & 13 & ATGTTGCCCAGGCTTGTCTC & CACCATTGCTTTCCAATGCG & 420 \\
\hline & 14 & GGATACAGCACTACCTCCTC & TCAGGAACGTCTTTGGACAG & 300 \\
\hline & 15 & GCTCAGTGTTGTCATGTTTC & GGAATCCATTGTAGAGAATG & 240 \\
\hline & 16 & TCGCCATCTGTTCTTCAAGT & AAAAGCACAACTCTTGGAGG & 269 \\
\hline
\end{tabular}

Abbreviations: MYOC, myocilin; OPTN, optineurin. 
Table 2 MYOC and OPTN sequence variants found in POAG patients and control Hispanics of Mexican Descent

\begin{tabular}{|c|c|c|c|c|c|c|c|}
\hline \multirow[b]{2}{*}{ Gene } & \multirow[b]{2}{*}{ Location } & \multirow[b]{2}{*}{ Sequence change } & \multirow[b]{2}{*}{ Codon change ${ }^{a}$} & \multirow[b]{2}{*}{$S N P I D$} & \multirow[b]{2}{*}{ Allele } & \multicolumn{2}{|c|}{ Minor allele frequency } \\
\hline & & & & & & $P O A G^{\mathrm{b}}$ & Control $^{\mathrm{C}}$ \\
\hline \multirow[t]{6}{*}{ MYOC } & Exon 1 & $G>A$ & $\mathrm{R} 7 \mathrm{H}$ & Unlisted & $A$ & 0.01 & 0.00 \\
\hline & Exon 1 & $G>A$ & R76K & rs2234926 & A & 0.11 & 0.09 \\
\hline & Exon 1 & $\mathrm{C}>\mathrm{T}$ & $\mathrm{G} 122 \mathrm{G}$ & Unlisted & $\mathrm{T}$ & 0.01 & NA \\
\hline & Exon 3 & $\mathrm{G}>\mathrm{T}$ & T285T & Unlisted & $\mathrm{T}$ & 0.01 & NA \\
\hline & Exon 3 & $\mathrm{~T}>\mathrm{C}$ & Y347Y & rs61730974 & C & 0.02 & NA \\
\hline & Exon 3 & $A>G$ & K398R & rs56314834 & $\mathrm{G}$ & 0.01 & 0.00 \\
\hline \multirow[t]{4}{*}{ OPTN } & Intron 2 & $4 \mathrm{bp}(\mathrm{ACAC})$ & c.374-194_374-193insACAC & rs67406260 & ACAC & 0.30 & 0.31 \\
\hline & Exon 4 & $G>A$ & T34T & rs2234968 & $A$ & 0.27 & 0.26 \\
\hline & Exon 5 & $\mathrm{~T}>\mathrm{A}$ & M98K & rs11258194 & $A$ & 0.03 & 0.03 \\
\hline & Intron 6 & $\mathrm{~T}>\mathrm{C}$ & c. $553-5 \mathrm{~T}>\mathrm{C}$ & rs2244380 & C & 0.10 & 0.13 \\
\hline
\end{tabular}

Abbreviations: NA, not applicable; POAG, primary open-angle glaucoma; SNP, single-nucleotide polymorphism.

Control individuals were not genotyped across amplicons in which only previously reported and consistently neutral polymorphisms were found.

athe sequence files used to number residues were NM 000261.1 for MYOC and NM_001008211.1 for OPTN.

aThe sequence files used to number residues were NM_O00261.1 for MYOC and NM_001008
b88 POAG cases were sequenced across all myocilin (MYOC) and optineurin (OPTN) exons.

c93 controls were sequenced across all MYOC and OPTN exons except for those which only contained known polymorphisms in the cases.

$(P<0.0001)$. Of the POAG cases for which highest IOP measurements had been obtained for both eyes, a majority, 59\%, had IOP below $22 \mathrm{~mm} \mathrm{Hg}$.

\section{Sequencing results}

MYOC. A single novel nonsynonymous coding variant, $\mathrm{R} 7 \mathrm{H}$, was identified in MYOC. This variant was identified in one allele of a single affected individual with an age at onset of 52 years. Five additional coding variants were identified: three synonymous (G122G, T285T and Y347Y) and two nonsynonymous (R76K and K398R; Table 2). None of these variants had allele frequencies that differed significantly between cases and controls $(P>0.05)$.

OPTN. Four variants were identified in the optineurin (OPTN) gene: two intronic (c.374-194_374-193insACAC and c.553-5T >C), one synonymous (T34T) and one nonsynonymous (M98K). None of these variants were significantly associated with POAG in this population $(P>0.05)$.

\section{DISCUSSION}

Two previous studies examining eye disease in Hispanics in the southwestern United States found that POAG is highly prevalent in Hispanics. Disease-associated MYOC variants have been reported in $3-5 \%$ of POAG cases from many different populations, ${ }^{11,13-15}$ including the Brazilian and Spanish populations. ${ }^{17,19}$ This study was undertaken to determine the role of MYOC and OPTN variants in POAG in the Hispanic population of Mexican descent. Sequence analysis of 88 POAG patients revealed six MYOC and four OPTN sequence variants. Of the six MYOC variants, five (G122G, T285T, Y347Y, R76K and K398R) were previously reported as neutral variants. ${ }^{13,24,25}$ Our results also indicate that these variants are neutral as they are not found at significantly different frequencies in cases and controls. It is unclear whether the novel MYOC variant, $\mathrm{R} 7 \mathrm{H}$ in exon 1, is a diseaseassociated variant because it was found as a heterozygous change in only one individual. The individual with the $\mathrm{R} 7 \mathrm{H}$ variant had an age of onset of POAG of 52 years and a highest recorded IOP of $38 \mathrm{~mm} \mathrm{Hg}$, which would be consistent with the phenotype of a myocilin-associated form of POAG in which IOP is almost always significantly elevated. However, the majority of disease-associated variants in myocilin are found in exon 3, although disease-associated variants have been reported in exon $1{ }^{26} \mathrm{~A}$ study of Brazilian patients by Povoa et al. ${ }^{17}$ found the MYOC variant Cys433Arg in $3.1 \%$ of POAG patients and in $5.2 \%$ of POAG patients with a family history of the disease. In a similar study on 110 Spanish subjects with POAG, Lopez-Martinez et al. found that $2.7 \%$ had causative MYOC variants, including Gln368Stop, Ala445Val and Tyr479His. ${ }^{19}$ We did not find these variants from either study in our research. Although MYOC mutations are consistently found in a low percentage of subjects with POAG in populations worldwide, we found no documented patho logical mutations in this Hispanic population of Mexican descent.

Variants in OPTN were originally identified in families with normal tension glaucoma. ${ }^{12}$ Two population-based studies found that $80 \%$ of POAG cases had normal IOPs on ascertainment. ${ }^{6,7}$ Despite this phenotypic characteristic, we were unable to find evidence that OPTN variants have a contributory role in POAG in this population. We found four sequence variants in OPTN. Two variants, T34T and c.553-5T $>\mathrm{C}$, have previously been reported to be neutral polymorphi sms. ${ }^{12,18,21,27,28}$ The M98K variant was initially reported to be associated with POAG risk, but the results of subsequent studies are conflicting. ${ }^{1,12,16,19,21,29-33}$ c.374-194_374-193insACAC has not been reported in the context of previous glaucoma studies, but is listed in dbSNP (http://www.ncbi.nlm.nih.gov/projects/SNP/). None of these OPTN variants, including M98K, were significantly associated with POAG in this population. Studies on other Hispanic populations also show a low prevalence of OPTN variants that cause POAG. LopezMartinez et al. failed to find OPTN variants that were significantly associated with POAG in an analysis of OPTN sequence variants in Spanish POAG patients. ${ }^{19}$ Similarly, a study conducted by CaixetaUmbelino et al., which examined the role of multiple OPTN variants in the development of POAG in a Brazilian population, did not find any association between the evaluated variants and POAG risk. ${ }^{18}$ Our findings also support the findings of these studies regarding the role of OPTN in POAG in populations of Hispanic descent.

This study is the first reported survey of MYOC and OPTN sequence variants in a Hispanic population of Mexican descent. Our results suggest that the contribution of these genes to the high prevalence of POAG in this population is small. These results should be confirmed with a larger sample size of this population. 


\section{ACKNOWLEDGEMENTS}

This work would have been impossible without the generous participation of POAG patients and their families. The study was supported by NIH grants R01EY013315 (MAH), R01 EY019126 (MAH) and R01 EY015543-04 (RRA).

1 Libby, R. T., Gould, D. B., Anderson, M. G. \& John, S. W. Complex genetics of glaucoma susceptibility. Annu. Rev. Genomics Hum. Genet. 6, 15-44 (2005).

2 Tielsch, J. M., Sommer, A., Katz, J., Royall, R. M., Quigley, H. A. \& Javitt, J. Racial variation in the prevalence of primary open-angle glaucoma: the Baltimore Eye Survey. JAMA 266, 369-374 (1991).

3 Quigley, H. A. Number of people with glaucoma worldwide. Br. J. Ophthalmol. 80, 389-393 (1996).

4 Quigley, H. A. \& Broman, A. T. The number of people with glaucoma worldwide in 2010 and 2020. Br. J. Ophthalmol. 90, 262-267 (2006).

5 Allingham, R. R., Liu, Y. \& Rhee, D. J. The genetics of primary open-angle glaucoma: a review. Exp. Eye Res. 88, 837-844 (2009).

6 Varma, R., Ying-Lai, M., Francis, B. A., Nguyen, B. B., Deneen, J., Wilson, M. R. et al. Prevalence of open-angle glaucoma and ocular hypertension in Latinos: the Los Angeles Latino Eye Study. Ophthalmology 111, 1439-1448 (2004).

7 Quigley, H. A., West, S. K., Rodriguez, J., Munoz, B., Klein, R. \& Snyder, R. The prevalence of glaucoma in a population-based study of Hispanic subjects: Proyecto VER. Arch. Ophthalmol. 119, 1819-1826 (2001).

8 Suzuki, Y., Iwase, A., Araie, M., Yamamoto, T., Abe, H., Shirato, S. et al. Risk factors for open-angle glaucoma in a Japanese population: the Tajimi Study. Ophthalmology 113, 1613-1617 (2006).

9 Sarfarazi, M. \& Rezaie, T. Optineurin in primary open angle glaucoma. Ophthalmol. Clin. North Am. 16, 529-541 (2003).

10 Rozsa, F. W., Shimizu, S., Lichter, P. R., Johnson, A. T., Othman, M. I., Scott, K. et al. GLC1A mutations point to regions of potential functional importance on the TIGR/ MYOC protein. Mol. Vis. 4, 20 (1998).

11 Stone, E. M., Fingert, J. H., Alward, W. L. M., Nguyen, T. D., Polansky, J. R., Sunden, S. L. F. et al. Identification of a gene that causes primary open angle glaucoma. Science 275, 668-670 (1997).

12 Rezaie, T., Child, A., Hitchings, R., Brice, G., Miller, L., Coca-Prados, M. et al. Adultonset primary open-angle glaucoma caused by mutations in optineurin. Science 295 , 1077-1079 (2002).

13 Wiggs, J. L., Allingham, R. R., Vollrath, D., Jones, K. H., De La Paz, M., Kern, J. et al. Prevalence of mutations in TIGR/Myocilin in patients with adult and juvenile primary open-angle glaucoma. Am. J. Hum. Genet. 63, 1549-1552 (1998),

14 Challa, P., Herndon, L. W., Hauser, M. A., Broomer, B. W., Pericak-Vance, M. A., Ababio-Danso, B. et al. Prevalence of myocilin mutations in adults with primary openangle glaucoma in Ghana, West Africa. J. Glaucoma. 11, 416-420 (2002).

15 Alward, W. L., Fingert, J. H., Coote, M. A., Johnson, A. T., Lerner, S. F., Junqua, D. et al. Clinical features associated with mutations in the chromosome 1 open-angle glaucoma gene (GLC1A). N Engl. J. Med. 338, 1022-1027 (1998).

16 Melki, R., Belmouden, A., Akhayat, O., Brezin, A. \& Garchon, H. J. The M98K variant of the OPTINEURIN (OPTN) gene modifies initial intraocular pressure in patients with primary open angle glaucoma. J. Med. Genet. 40, 842-844 (2003).
17 Povoa, C. A., Malta, R. F., Rezende, M. M., de Melo, K. F. \& Giannella-Neto, D. Correlation between genotype and phenotype in primary open angle glaucoma of Brazilian families with mutations in exon 3 of the TIGR/MYOC gene. Arq. Bras. Oftalmol. 69, 289-297 (2006).

18 Caixeta-Umbelino, C., de Vasconcellos, J. P., Costa, V. P., Kasahara, N., Della, P. M., de Almeida, G. V. et al. Lack of association between optineurin gene variants T34T, E50K, M98K, 691_692insAG and R545Q and primary open angle glaucoma in Brazilian patients. Ophthalmic Genet 30, 13-18 (2009).

19 Lopez-Martinez, F., Lopez-Garrido, M. P., Sanchez-Sanchez, F., Campos-Mollo, E., Coca-Prados, M. \& Escribano, J. Role of MYOC and OPTN sequence variations in Spanish patients with primary open-angle glaucoma. Mol. Vis. 13, 862-872 (2007).

20 Rozen, S. \& Skaletsky, H. Primer3 on the WWW for general users and for biologist programmers. Methods Mol. Biol. 132, 365-386 (2000).

21 Liu, Y., Akafo, S., Santiago-Turla, C., Cohen, C. S., Larocque-Abramson, K. R., Qin, X. et al. Optineurin coding variants in Ghanaian patients with primary open-angle glaucoma. Mol. Vis. 14, 2367-2372 (2008).

22 Liu, Y., Schmidt, S., Qin, X., Gibson, J., Hutchins, K., Santiago-Turla, C. et al. Lack of association between LOXL1 variants and primary open-angle glaucoma in three different populations. Invest Ophthalmol. Vis. Sci. 49, 3465-3468 (2008).

23 Kruglyak, L. \& Nickerson, D. A. Variation is the spice of life. Nat. Genet. 27, 234-236 (2001).

24 Melki, R., Idhajji, A., Driouiche, S., Hassani, M., Boukabboucha, A., Akhayat, O. et al. Mutational analysis of the Myocilin gene in patients with primary open-angle glaucoma in Morocco. Ophthalmic Genet. 24, 153-160 (2003).

25 Fingert, J. H., Heon, E., Liebmann, J. M., Yamamoto, T., Craig, J. E., Rait, J. et al. Analysis of myocilin mutations in 1703 glaucoma patients from five different populations. Hum. Mol. Genet. 8, 899-905 (1999).

26 Hewitt, A. W., Mackey, D. A. \& Craig, J. E. Myocilin allele-specific glaucoma phenotype database. Hum. Mutat. 29, 207-211 (2008).

27 Forsman, E., Lemmela, S., Varilo, T., Kristo, P., Forsius, H., Sankila, E. M. et al. The role of TIGR and OPTN in Finnish glaucoma families: a clinical and molecular genetic study. Mol. Vis. 9, 217-222 (2003).

28 Ayala-Lugo, R. M., Pawar, H., Reed, D. M., Lichter, P. R., Moroi, S. E., Page, M. et al. Variation in optineurin (OPTN) allele frequencies between and within populations. Mol. Vis. 13, 151-163 (2007).

29 Ariani, F., Longo, I., Frezzotti, P., Pescucci, C., Mari, F., Caporossi, A. et al. Optineurin gene is not involved in the common high-tension form of primary open-angle glaucoma. Graefes Arch. Clin. Exp. Ophthalmol. 244, 1077-1082 (2006).

30 Baird, P. N., Richardson, A. J., Craig, J. E., Mackey, D. A., Rochtchina, E. \& Mitchell, P. Analysis of optineurin (OPTN) gene mutations in subjects with and without glaucoma: the Blue Mountains Eye Study. Clin. Experiment. Ophthalmol. 32, 518-522 (2004).

31 Hauser, M. A., Sena, D. F., Flor, J., Walter, J., Auguste, J., LaRocque-Abramson, K. et al. Distribution of optineurin sequence variations in an ethnically diverse population of low-tension glaucoma patients from the United States. J. Glaucoma. 15, 358-363 (2006).

32 Wiggs, J. L., Auguste, J., Allingham, R. R., Flor, J. D., Pericak-Vance, M. A., Rogers, K. et al. Lack of association of mutations in optineurin with disease in patients with adultonset primary open-angle glaucoma. Arch. Ophthalmol. 121, 1181-1183 (2003).

33 Alward, W. L., Kwon, Y. H., Kawase, K., Craig, J. E., Hayreh, S. S., Johnson, A. T. et al. Evaluation of optineurin sequence variations in 1,048 patients with open-angle glaucoma. Am. J. Ophthalmol. 136, 904-910 (2003). 